August 9, 2018

\title{
Supersymmetry, Naturalness, and Dynamical Supersymmetry Breaking ${ }^{\star}$
}

\author{
Michael Dine and Douglas A. MacIntire \\ Santa Cruz Institute for Particle Physics \\ University of California, Santa Cruz, CA 95064
}

\begin{abstract}
Models with dynamical supersymmetry breaking have the potential to solve many of the naturalness problems of hidden sector supergravity models. We review the argument that in a generic supergravity theory in which supersymmetry is dynamically broken in the hidden sector, only tiny Majorana masses for gauginos are generated. This situation is similar to that of theories with continuous Rsymmetries, for which Hall and Randall have suggested that gluino masses could arise through mixings with an octet of chiral fields. We note that in hidden sector models, such mixing can only occur if the auxiliary D field of a $U(1)$ gauge field has an expectation value. This in turn gives rise to a catastrophically large FayetIliopoulos term for ordinary hypercharge. To solve this problem it is necessary to unify hypercharge at least partially in a non-Abelian group. We consider, also, some general issues in models with continuous or discrete $\mathrm{R}$ symmetries, noting that it may be necessary to include $S U(2)$ triplet fields, and that these are subject to various constraints. In the course of these discussions, we consider a number of naturalness problems. We suggest that the so-called " $\mu$-problem" is not a problem, and point out that in models in which the axion decay constant is directly related to the SUSY breaking scale, squarks, sleptons and Higgs particles generically acquire huge masses.
\end{abstract}

Submitted to Physical Review D.

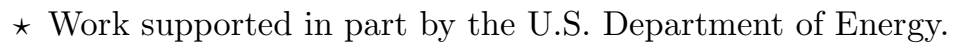




\section{Introduction}

Hidden sector supergravity models provide a framework in which to understand how various types of soft supersymmetry breaking couplings might arise at low energies. Unfortunately, none of the models which have been constructed to date are at all compelling. None are beautiful, and all suffer from serious naturalness problems. The most severe of these is the problem of the cosmological constant. Others include too large flavor changing neutral currents and neutron electric dipole moment, the existence of a large hierarchy, put in by hand, and the need to omit from the lagrangian numerous couplings permitted by symmetries, involving both visible sector and hidden sector particles.

About the cosmological constant, we will have nothing new to say here. We will have to simply assume that this problem is solved by some mechanism which does not too drastically alter the low energy structure of the theory. One might hope to find conventional field-theoretic explanations for the other questions. If this is the case, these might have phenomenologically interesting consequences, leading to predictions concerning the spectrum of supersymmetric particles. About flavor changing currents and CP, for example, it has been noted elsewhere ${ }^{[1]}$ that this problem might be resolved if the gauginos are the most massive supersymmetric particles. The problems of obtaining a large hierarchy and of the omission of numerous couplings in the hidden sector might be resolved if supersymmetry is dynamically broken. ${ }^{[2-4]}$ The need to omit various visible sector couplings, on the other hand, might be resolved by the recent suggestion of Hall and Randall that one impose an R symmetry on the theory. ${ }^{[5]}$

Theories with dynamical supersymmetry breaking (DSB) have been known

for some time. ${ }^{[2-4]}$ DSB has the potential to explain large hierarchies, and the known examples have the virtue that it is not necessary to omit couplings allowed by symmetries. However, if one proceeds in the most straightforward way to build models based on these, one runs into difficulties. The most severe of these concerns masses for gauginos, which turn out to be extremely small. Of course, models with $\mathrm{R}$ symmetries also are in danger of yielding small (zero) gaugino masses. In the latter case, Hall and Randall have proposed that the problem can be solved by adding an octet of chiral fields to the low energy theory; these particles combine 
with the gauginos to gain mass. This mechanism is a potential solution to the problems of DSB as well. We will see, however, that in either case this mechanism can operate only if the auxiliary $\mathrm{D}$ field of a $U(1)$ gauge field in the hidden sector acquires a large expectation value. This in turn raises the danger of a large FayetIliopoulos term for hypercharge. ${ }^{[6]}$ One way (possibly the only way) to forbid such a term is to unify hypercharge in a non-Abelian group at some scale. We will see that the natural scale for breaking this additional symmetry is the hidden sector scale.

While DSB may resolve some questions of naturalness in the hidden sector, it is still usually necessary to forbid certain visible sector couplings. For example, in the minimal supersymmetric standard model, one must forbid a large mass term in the superpotential for the two Higgs doublets, but one must have a soft breaking mass term involving both doublets. One can, as in the case of the unwanted hidden sector couplings, simply suppose that the unwanted superpotential terms are not present at tree level and then invoke non-renormalization theorems. Superstring theory suggests that such a possibility might not be unreasonable. Hall and Randall $^{[5]}$ have recently considered an alternative possibility, noting that continuous $\mathrm{R}$ symmetries can forbid such terms.

The rest of this paper is organized as follows. In the next section, we will briefly review some features of models with dynamically broken supersymmetry. In section 3, we will consider what happens when such models are coupled to supergravity. We will recall the general arguments that majorana masses for gauginos in such theories must be small, and illustrate them with a one loop calculation. This calculation is rather subtle; a conventional treatment, such as has been applied to supergravity theories in the past, ${ }^{[7]}$ gives a large mass. It turns out, however, that the Feynman diagrams contributing to the gaugino mass require careful regularization, and that in the end these masses are small. We comment on the implications of these results for more conventional theories.

In section 4 , we consider the effect of adding an octet to a theory in which majorana masses for gauginos are small. We first consider the problems associated with D terms, and possible solutions. We then consider models with dynamical supersymmetry breaking in which either gauge interactions or supergravity is the "messenger" of supersymmetry breaking. It does not appear too difficult to build 
realistic models of this type.

In section 5 we consider some aspects of models in which majorana masses for gauginos are forbidden by continuous or discrete $\mathrm{R}$ symmetries. We point out, first, that in supergravity theories, if one insists on cancelling the cosmological constant in the effective lagrangian, only a discrete $Z_{2} \mathrm{R}$ symmetry can survive to low energies. We argue, however, that given our poor understanding of the cosmological constant problem, and given all of the naturalness problems of supersymmetric theories, such symmetries are still worthy of study. As noted by Hall and Randall, in such theories, in addition to an octet of chiral fields, it may be necessary to have still other fields to avoid very light states in the neutralino sector. These authors considered the possible addition of a gauge singlet superfield. We show that such a singlet is unnatural, in the sense that in almost any conceivable scheme for supersymmetry breaking, it has unacceptable properties. We note that the corresponding problems do not arise for $S U(2)$ triplet fields, and consider some aspects of such models, including the spectrum and the question of the $\rho$ parameter. We find that such schemes typically predict that there should be new particles with masses below $M_{Z}$.

Our conclusions are presented in section 6 . Here we comment on possible connections of axion physics and supersymmetry. In particular, it is remarkable that both of these require a scale of around $10^{11} \mathrm{GeV}$, and a number of authors ${ }^{[8,9]}$ have speculated on possible connections between them. We point out that generically, if the Peccei-Quinn symmetry is broken by vev's in the hidden sector, squark, slepton and Higgs masses tend to be of order the intermediate scale. 


\section{Dynamical Supersymmetry Breaking}

Witten was perhaps the first to appreciate the possible importance of dynamical supersymmetry breaking and to clearly formulate the problem. ${ }^{[10]}$ He stressed that dynamical supersymmetry breaking was likely to give rise to large hierarchies. Because of the non-renormalization theorems, ${ }^{[1]]}$ supersymmetry, if unbroken at tree level, remains unbroken to any finite order in perturbation theory. However, he pointed out that the proofs of the non-renormalization theorems are firmly based on perturbation theory. Thus one can hope to find effects smaller than any power of the coupling constant which give rise to supersymmetry breaking. Witten went on to prove that many theories do not break supersymmetry dynamically ${ }^{[12]}$ However, chiral gauge theories did not yield to this analysis.

In a series of papers, it was in fact shown that supersymmetry is sometimes dynamically broken in four dimensions. ${ }^{[2-4]}$ First it was observed that nonperturbative breakdown of the non-renormalization theorems is common - almost generic. The basic point is illustrated by an $S U(2)$ gauge theory with a single massless flavor, i.e. containing two chiral doublets, $Q$ and $\bar{Q}$. At the classical level, this theory has a continuum of physically inequivalent vacuum states. Essentially these are the states with $Q=\bar{Q}=\left(\begin{array}{l}0 \\ v\end{array}\right)$. For non-zero $v$, the gauge symmetry is completely broken, and the gauge bosons are massive. The effective coupling in a given vacuum is $g(v)$, since the gauge boson masses are of order $v$, and all momentum integrals are cut off in the infrared at this scale. As the theory is asymptotically free, by choosing $v$ large enough the theory may be made as weakly coupled as one wishes. In each of these states there is one massless chiral field. This field can be written in a gauge-invariant way as $\Phi=\bar{Q} Q$. Expanding the fields $Q$ and $\bar{Q}$ in small fluctuations about their vacuum expectation values, the term linear in the fluctuations is the massless state. The problem, then, is to understand the properties of the effective low energy theory containing $\Phi$ only, and in particular to determine whether this theory possesses a superpotential for $\Phi$. Symmetry considerations restrict the superpotential to be of the form

$$
W=\frac{\Lambda^{5}}{\Phi}
$$


Here $\Lambda$ is the scale of this $\mathrm{SU}(2)$ theory, and again, this expression should be understood by expanding $\Phi$ in small fluctuations about a particular ground state.It is straightforward to show that a single instanton generates the various component interactions implied by this superpotential. This analysis immediately generalizes to theories with gauge group $S U(N)$ and $N-1$ flavors. Adding small mass terms, one finds that all of these results are consistent with Witten's analysis of dynamical supersymmetry breaking. Minimizing the full superpotential yields $N$ gauge-inequivalent ground states, in agreement with Witten's computation of the index. By other methods, one can show that a superpotential is generated in many other theories.

While these examples illustrate that the non-renormalization theorems do indeed break down non-perturbatively, they do not lead to a particularly interesting phenomenology. Without mass terms, the potential for the field $\Phi$ falls rapidly to zero for large $\Phi$. Thus the model has at best a cosmological interpretation. The basic problem is that for large expectation values of the fields, the theories become more weakly coupled and any potential which is generated non-perturbatively must tend to zero. Adding mass terms to the theory eliminates the "flat directions" which exist classically in the potential, but in this case the full theory has supersymmetric ground states. A general criterion for obtaining supersymmetry breaking with a good ground state was suggested in ref. 3. Suppose a theory has, classically, no flat directions in its potential. At the same time suppose that the theory possesses a continuous global symmetry which is broken in the true vacuum. In such circumstances supersymmetry is almost certainly broken. For, if it were not, the goldstone bosons of the broken symmetry would have scalar partners which would have no potential. However, in this case their expectation values would not be fixed and there would be flat directions, contradicting the original assumption.

This argument is heuristic, and one can imagine a variety of loopholes. However, a number of models were studied in ref. 4 satisfying these criteria, and shown to break supersymmetry. The simplest is a theory with gauge group $S U(3) \times S U(2)$, with chiral fields $Q, \bar{U}, \bar{D}$ and $L$, transforming as $(3,2)$, two $(\overline{3}, 1)$ 's and $(1,2)$, respectively under the group. In addition to the gauge interactions, to eliminate the flat directions it is necessary to include a superpotential 


$$
W=\lambda Q \bar{Q} L .
$$

If $\lambda$ is small, one can first determine the superpotential generated by instantons (as in equation (2.1)), and then treat the tree level superpotential (equation (2.2)) as a small correction. Minimimizing the resulting potential, one finds that supersymmetry is broken. If the scale of the $S U(3), \Lambda_{3}$, is larger than that of $S U(2)$, $\Lambda_{2}$, one finds that at the minimum

$$
Q=\left(\begin{array}{ccc}
a & 0 & 0 \\
0 & b & 0
\end{array}\right) \quad \bar{Q}=\left(\begin{array}{c}
\bar{U} \\
\bar{D}
\end{array}\right)=Q \quad L=\left(\begin{array}{c}
\sqrt{a^{2}-b^{2}} \\
0
\end{array}\right)
$$

where $a=1.286 \Lambda_{3} / \lambda^{\frac{1}{7}}, b=1.249 \Lambda_{3} / \lambda^{\frac{1}{7}}$, and the vacuum energy is $E=$ $3.593 \lambda^{\frac{10}{7}} \Lambda_{3}^{4}$.

Other models can be analyzed along similar lines. Another example of interest is an $\mathrm{SU}(5)$ theory with a single $\overline{5}$ and 10 . In this theory, there is no classical superpotential which one can write. Even so, the theory has no flat directions. Using 't Hooft anomaly conditions one can argue that the non-anomalous global symmetry of the model must be broken, and that as a result supersymmetry is broken.

\section{Coupling to Supergravity}

We would like to consider a theory of this type as a candidate for the hidden sector of a supergravity model. As a concrete example, we take the $S U(3) \times$ $S U(2)$ model described in the previous section; however, our considerations below generalize almost trivially to other theories. We assume that, apart from some possible superheavy $\left(\mathcal{O}\left(M_{P}\right)\right.$ or $\left.\mathcal{O}\left(M_{G U T}\right)\right)$ fields, no other fields transform under the $S U(3) \times S U(2)$ gauge symmetry of the hidden sector (these groups should not be confused with the $S U(3) \times S U(2) \times U(1)$ symmetry in the visible sector; they

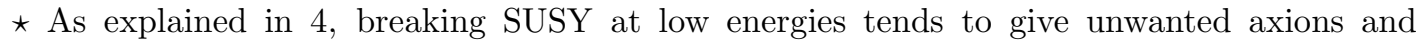
Goldstone bosons.
} 
represent additional gauge interactions). Thus, taking the characteristic scale of the hidden sector, $M_{\text {int }}$, to be $M_{\text {int }} \sim 10^{11} \mathrm{GeV}$, the dynamics described in the previous section are unaffected: supersymmetry is broken in this sector at a scale of order $M_{i n t}$; various fields acquire expectation values and masses of order $M_{i n t}$, and there are some (pseudo) Goldstone fields with decay constants of this order.

The analysis of scalar masses is similar to the case of more conventional hidden sector supergravity models. The problem comes when one attempts to compute gaugino masses. There is a simple argument that any Majorana masses for gauginos in such theories must be extremely small. In discussing physics at scales above $m_{3 / 2}$, it should be possible to integrate out Planck (and GUT) scale physics, obtaining a (locally) supersymmetric effective lagrangian. The usual supergravity lagrangian is specified by three functions. Only the function $f\left(\phi_{i}\right)$, which describes the coupling of the chiral fields to the gauge multiplets, is relevant to the question of gaugino mass through a term in the lagrangian:

$$
\mathcal{L} \sim \int d^{2} \theta f(\phi) W^{\alpha} W^{\alpha}
$$

where $f$ is a holomorphic function of the scalar fields. On the other hand, in all of the models of dynamical supersymmetry breaking presently known, all of the hidden sector fields, $Z_{i}$, carry charges under the various gauge symmetries. Thus $f$ is necessarily at least quadratic (and in fact is generally cubic) in fields. Thus one expects its coefficient to be suppressed by at least two powers of $M_{P}$. If this is the case, local supersymmetry implies that gaugino masses will be extremely small (of order $\mathrm{eV}$ or smaller).

However, if one simply computes the gaugino masses in these models using the naive Feynman rules, one seems to find much larger answers. For example, suppose that, in addition to a hidden sector of the type we have described in the previous section, the model possesses a heavy color octet, $O$, of chiral fields of mass $M$. Then at one loop there is a diagram contributing to the gluino mass, quite similar to the types of diagrams considered in ref. 7. In particular, there is a (non-vanishing) term in the lagrangian of the form $m_{3 / 2} M O^{2}$, where $O$ is the scalar component of the octet. Then the diagram of fig. 1 gives a non-zero mass 
for the gluino of order

$$
m_{\lambda} \sim \frac{\alpha_{3}}{\pi} \frac{M_{i n t}^{2}}{M_{P}}
$$

Notice, in particular, that this expression is independent of the mass of the octet. If correct, this would be a wonderful result, since it would give rise to a gluino mass of order $100 \mathrm{GeV}$ of so. Not surprisingly, in view of our general argument, this result is not supersymmetric.

The problem with this calculation is most easily illustrated with a well-studied model, the "Polonyi model." This theory contains a hidden sector consisting of only one singlet chiral field, $Z$, with superpotential

$$
W=M_{i n t}^{2}(Z+\beta)
$$

Assuming that the Kahler potential is simply quadratic in $Z$, the minimum of the potential occurs for

$$
Z=(\sqrt{3}-1) M
$$

where $M=\frac{M_{p}}{\sqrt{8 \pi}}$; in these equations, in order that the cosmological constant vanish at the minimum of the potential, $\beta=(2-\sqrt{3}) M$. Because $Z$ is a gauge singlet, there should be no problem obtaining a gaugino mass in this model, since any $f$ which is, say, a polynomial in $Z$ will yield $m_{\lambda} \sim m_{\frac{3}{2}}$. Indeed, if one now adds to this model the heavy octet, $O$, above, one generates a gluino mass at one loop; ${ }^{[7]}$ proceeding as before, the diagram of fig. 1 yields

$$
m_{\lambda}=\frac{3 g^{2}}{4 \pi^{2}}(2-\sqrt{3}) e^{\frac{(\sqrt{3}-1)^{2}}{2}} m_{\frac{3}{2}}
$$

with $m_{3 / 2} \sim \frac{M_{i n t}^{2}}{M_{p}}$. Again we find a mass of the order of $100 \mathrm{GeV}$.

Now we would expect that at energy scales below the mass of the heavy octet, $M_{O}$, the system would still be described by a locally supersymmetric effective lagrangian, including the usual light fields and the hidden sector fields. In particular, the gluino mass could be understood as arising from the function $f$ of this theory, 
through the term in the supergravity lagrangian:

$$
\mathcal{L} \sim \frac{1}{4} e^{-G / 2} G^{l}\left(G^{-1}\right)_{l}^{k} f_{\alpha \beta k}^{*} \lambda^{\alpha} \lambda^{\beta}
$$

In the above, $f_{\alpha \beta k}^{*}$ is the derivative of $f_{\alpha \beta}^{*}$ with respect to $Z$, and the existence of a gluino mass implies that $f$ is a function of $Z$. If this is the case, on the other hand, we expect to find couplings of $Z$ to $F_{\mu \nu}^{2}$ and $F \tilde{F}$ through the terms:

$$
\mathcal{L} \sim-\frac{1}{4} \operatorname{Re} f_{\alpha \beta} F_{\mu \nu}^{\alpha} F^{\mu \nu \beta}+\frac{1}{4} i \operatorname{Im} f_{\alpha \beta} F_{\mu \nu}^{\alpha} \tilde{F}^{\mu \nu \beta}
$$

However, at one loop, using the lagrangian of refs. 13, these couplings vanish!

To see this, consider the coupling of the pseudoscalar part of $Z$ to the octet fermions. This coupling is proportional to $\partial_{\mu} Z \bar{O} \gamma^{\mu}\left(1-\gamma_{5}\right) O$. One can attempt to compute the coupling of the imaginary part of $Z$ to $F \tilde{F}$ at one loop arising from this term. But this calculation is identical to the famous calculation of the chiral anomaly, and is subject to the same ambiguities. For example, it is well known that if one uses, say, a Pauli-Villars regulator, the $F \tilde{F}$ coupling vanishes in this case. Indeed, the result of this computation, as in the case of the gaugino mass above, is independent of the mass of the particle running in the loop, and so is canceled by the regulator diagram. Clearly supersymmetry requires that one use the same sort of regulator for all of the diagrams. But we have seen that the gluino mass is independent of the mass of the heavy particle running in the loop, so adding the Pauli-Villars term will give zero! Correspondingly, alternative choices of (supersymmetric) regulators will give different results for the gluino mass. However, in the case of the hidden sector with DSB, it is clear from our original symmetry arguments that any gauge- and supersymmetric regulator will give zero for the gaugino mass.

Thus simply using a theory with dynamical supersymmetry breaking as a conventional hidden sector model yields unacceptable results. In the following section, we consider an alternative approach.

\footnotetext{
$\star$ We are assuming, here, that the lagrangian given in ref. 13 is the most general one consistent with local supersymmetry, up to terms with two derivatives.
} 


\section{Models with Octets}

Majorana masses for gauginos are also forbidden in theories with an exact $\mathrm{R}$ symmetry at low energies. Following Hall and Randall, it is natural to attempt to build models with light color octet chiral fields, and to allow them to mix with the gluinos. In this section, we will consider some general issues in models of this kind (with either DSB and/or exact $\mathrm{R}$ symmetries).

As stressed by these authors, a mass term mixing the gluino and the octet fermions is one of the allowed soft breaking terms of supersymmetry. It is interesting to ask, on the other hand, how such a term might arise in the framework of hidden sector models. Consider, first, the case of hidden sector models with global supersymmetry. (It is convenient to consider this case because it is easy to write down globally supersymmetric effective actions). In such theories, above the scale of weak interactions (the scale of supersymmetry breaking in the visible sector of the theory), it is possible to describe the theory by a supersymmetric effective action. ${ }^{[6]}$ Then supersymmetry breaking is the statement that, below the breaking scale, the auxiliary $(F)$ component of some chiral superfield(s), $Z$, is non-vanishing, as well, possibly, as the auxiliary $(D)$ components of some gauge fields. In such theories, masses for the scalar components of observable fields (denoted by $\phi$ ), arise through operators of the type $\int d^{4} \theta Z^{\dagger} Z \phi^{\dagger} \phi$; Replacing $Z\left(F_{Z}\right)$ by its expectation value immediately yields scalar masses. On the other hand, terms which mix the fermionic components of the octet, $O$, with the gluinos can only arise provided the theory contains a $U(1)$ gauge field, $\tilde{V}$, whose auxiliary component, $\tilde{D}$, has an expectation value. ${ }^{\dagger}$ Then the desired mixing can arise through the operator

$$
\mathcal{L}_{\lambda}=\frac{1}{M} \int d^{2} \theta \tilde{W}_{\alpha} W_{\alpha}^{a} O^{a}
$$

Of course, this $U(1)$ cannot be ordinary hypercharge. But the large vev of $\tilde{D}$ raises the specter of a large Fayet-Iliopoulos term for $D^{Y}$. The dimension four

$\dagger$ This does not occur in the $S U(3) \times S U(2)$ model discussed earlier. There we can gauge a $U(1)$. However, it is is necessary to include an additional field to cancel anomalies. It turns out that the sign of the charge of this field is such that its expectation value gives a vanishing expectation value for $\tilde{D}$. We know of no reason for this to be true in general, however. 
operator $\int d^{2} \theta W_{\alpha}^{Y} \tilde{W}^{\alpha}$ gives a Fayet-Iliopoulos term of order $\langle\tilde{D}\rangle$. Such a coupling implies a large negative mass-squared for scalars carrying hypercharge (of order $M_{\text {int }}^{2}$ ), and potentially leads to an enormous breaking of hypercharge. One possible way to avoid this problem is to unify hypercharge into a non-Abelian group, broken only at some scale well below $M_{p}$. For example, many authors, motivated by string theory, have considered the possibility that down to some scale there is an unbroken $S U(3)_{c} \times S U(3)_{L} \times S U(3)_{R}$ symmetry. In such a case, the Fayet-Iliopoulos term could be highly suppressed. In fact, one can even avoid the problem if hypercharge is a sum of a $U(1)$ generator and a non-Abelian generator. In such a case, it can be natural for some scalar field to gain a large vev, breaking some of the gauge symmetry and leaving ordinary hypercharge.

These considerations can be immediately extended to the case of local supersymmetry. If one examines the lagrangian of ref. 13, one can see that there is only one term which gives rise to a Dirac mass term mixing gauginos and matter fields, and this is only non-vanishing if there is an expectation value for $\tilde{D}$.

In theories with dynamical supersymmetry breaking, having obtained a sufficiently large gluino mass, we have more or less phenomenologically acceptable models. One still must check the neutralino sector. If the superpotential contains a term $m H \bar{H}$, with $m \sim m_{3 / 2}$, the only light neutralino is the photino. We will argue later that a term of this size will arise automatically in many circumstances. The photino may gain a small mass from loops of light fields, but it may be necessary to add additional light fields in order to obtain an acceptable phenomenology.

$\ddagger$ As an example, one can consider a set of fields with the quantum numbers of a 27 of $E_{6}$, and suppose that the unbroken group is $S U(2)_{L} \times S U(2)_{R} \times U(1) \times U(1)$. Suppose that, apart from the Fayet-Iliopoulos term for the $U(1)$, all fields have positive soft-breaking mass terms, of order $m_{3 / 2}^{2}$, except for the two $S U(3) \times S U(2) \times U(1)$ singlets, which have negative mass-squared terms. Then it is easy to check that there is a local minimum of the potential at which the surviving gauge symmetry is $S U(3) \times S U(2) \times U(1)$. 


\section{Models with Continuous R symmetries}

Our remarks in the previous section apply to models with dynamical supersymmetry breaking and to theories with unbroken continuous $\mathrm{R}$ symmetries. In both types of models, the desired mixing arises if an auxiliary D field has a nonzero vev, and one must insist on at least a partial unification of hypercharge in a non-Abelian group to avoid Fayet-Iliopoulos terms. In this section, we consider some further issues associated with $\mathrm{R}$ symmetries. Such theories have previously been carefully considered by Hall and Randall. ${ }^{[5]}$ These authors assumed that the symmetry was continuous. The Higgs fields were assigned $R=0$, while quark and lepton superfields were assigned $R=1$. In order to obtain a gluino mass, they required that their models contain a color octet chiral field with $R=0$; they then noted that $\lambda^{a} \psi_{O}^{a}$, where $\psi_{O}$ is the fermionic component of $O$, is one of the allowed soft breaking terms. Hall and Randall also observed that if one does not add additional fields, the model possesses, at tree level, a massless photino and a massless higgsino. At tree level, one can suppress the coupling of the massless Higgsino to the Z, however one predicts too light a Higgs. As a result, these authors considered theories with an additional singlet field. We will argue shortly that in almost any scenario for supersymmetry breaking, this is likely to lead to difficulties; instead one needs to add $S U(2)$ triplet fields. Hall and Randall have recently pointed out that once one loop corrections are accounted for, it may not be necessary to include additional fields at all. The point is that the large radiative corrections to the Higgs mass due to top quark loops which have been discovered recently ${ }^{[14]}$ can avoid the light Higgs problem, provided the top quark is heavy enough. ${ }^{[15]}$ Of course, dynamical supersymmetry breaking could operate in the framework of such models as well.

We would like to explore some aspects of models of this type. First, there are some questions of "philosophy" and naturalness which must be addressed. For most particle theorists, continuous global symmetries are anathema, and this might be viewed as an objection to the work of ref. 5. However, in order to implement the program of these authors, it is not necessary that the $\mathrm{R}$ symmetry be continuous; it can in fact be discrete. Discrete $R$ symmetries have a different status. For example, they arise frequently in string theory, where they are usually (possibly 
always) discrete gauge symmetries. For suitable $Z_{N}$, a discrete $Z_{N} \mathrm{R}$ symmetry has consequences very similar to that of a continuous $\mathrm{R}$ symmetry.

For both discrete or continuous $\mathrm{R}$ symmetries, however, there is a puzzle. Supersymmetry, if it exists, is a local symmetry. Thus the underlying theory must be a supergravity theory. In an $N=1$ supergravity theory, supersymmetry breaking with vanishing energy at the minimum of the potential requires that the superpotential have a non-zero expectation value. But such an expectation value necessarily breaks any R-invariance (apart from $Z_{2}$ symmetries). In simple models, this breaking of $\mathrm{R}$ invariance tends to be large, and, for example, large Majorana mass terms for gauginos are generated. Still, given our lack of understanding of the cosmological constant problem, the possibility of an unbroken R-invariance seems worthy of investigation.

On the other hand, we would like to reconsider the motivations for considering R-symmetric theories given in ref. 5. These authors argue that such symmetries would improve the "naturalness" properties of supersymmetric theories. For example, they would forbid a term in the superpotential of the form $\mu H_{1} H_{2}$, where $H_{i}$ denote the two Higgs doublets. This argument is not particularly compelling. From string theory, for example, we know that it is plausible to have massless Higgs doublets at tree level and to any finite order in perturbation theory. The question, then, is how large is $\mu$ once one takes into account supersymmetry breaking. The situation is most easily described in global supersymmetry. There, if the hidden sector contains some fields, $Z_{i}$, with non-vanishing $F$-components, $F \sim M_{\text {int }}^{2} \sim m_{W} M$, then $\mu$ is generated by operators of the form

$$
\mathcal{L}_{\mu}=\frac{1}{M} \int d^{4} \theta Z^{\dagger} H_{1} H_{2}
$$

Replacing the chiral field $Z$ by its vacuum expectation value $Z=\ldots \theta^{2}\langle F\rangle$ gives $\mu=\frac{\langle F\rangle}{M} \sim m_{W}$. A number of authors have noted that these couplings can arise in loops. In supergravity theories, they generically arise at tree level. For example, in an $S U(5)$ theory in which a 24 couples to Higgs in the superpotential, in such

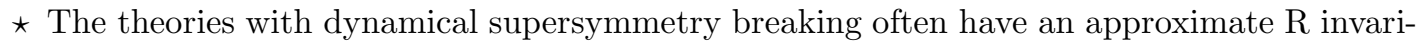
ance in the low energy theory, even after cancelling the cosmological constant, but this does not help with the basic problems of naturalness.
} 
a way that the Higgs mass vanishes as $m_{3 / 2} \rightarrow 0$, supersymmetry breaking shifts the 24 vev by an amount of order $m_{3 / 2}$, giving rise to $\mu \sim m_{3 / 2}$. Thus, in a generic theory, the " $\mu$-problem" does not appear to be a problem.

The question of motivation aside, models with $\mathrm{R}$ symmetry are quite interesting. Singlets, however, are likely to lead to difficulty in this context. The problem is that the dimension four term in the effective lagrangian,

$$
\mathcal{L}_{S}=\int d^{4} \theta Z^{\dagger} S
$$

gives rise, effectively, to a superpotential term

$$
W_{S}=\langle F\rangle S
$$

Because $F$ is generically so large, this term generally has disastrous consequences; for example, it leads to expectation values for Higgs doublets of order the intermediate scale. If one has, instead of singlets, some additional triplet fields, this problem does not arise. The corresponding " $\mu$ " term, as for Higgs fields, is of order $m_{W}$.

Actually, in models with dynamical supersymmetry breaking, in contrast to the more general case, this problem may be somewhat ameliorated. The point is, again, that the $Z_{i}$ 's are all charged under the hidden sector gauge symmetries, so it is necessary to go to higher dimension operators in order to find these $\mu$ terms. Explicit checks show that at one loop, such terms are indeed generated only with suitably small coefficients. Thus in this framework, models with singlets may make sense. However, it is of some interest to explore the case of models with triplets as well. This is rather straightforward extension of the work of ref. 5, which we now describe.

In the case of triplets, there are a number of phenomenological concerns. One has to insure that the triplet expectation values are small enough that the $\rho$ parameter is not significantly affected. Also, one must make sure that there are no particles so light that their effects would already have been observed at LEP. For definiteness, we will focus on the case where the $R$ symmetry is continuous.

As usual, we assign all ordinary matter fields R-charge zero; in other words, the chiral fields associated with the quarks and leptons are assigned $R=1$, while 
those associated with the Higgs are assigned $R$ charge zero. Gauginos have $R=+1$. We want to add an octet and a triplet field to the model. A moment's thought indicates that it is necessary to add at least two triplets to the model if one is to avoid massless particles. The problem is that in the neutralino sector, with only one triplet (taking, for a moment, the triplet to have $R=0$ ) there are two positively charged, left-handed fermions with $R=-1$, while there is only one with $R=+1$; similar problems arise in the other charge sectors. This problem can be solved if we include two triplets in the model, one with $R=2$, and one with $R=0$. These will be denoted by $\hat{T}$ and $\hat{T}^{\prime}$, respectively. The additional terms allowed in the superpotential are then

$$
W_{T}=G \hat{T}^{a} \hat{H}_{2} \epsilon \tau^{a} \hat{H}_{1}+B \hat{T}^{\prime a} \hat{T}^{a}
$$

The scalar potential generated by this superpotential is:

$$
\begin{gathered}
V=\left(\frac{g^{\prime 2}+g^{2}}{32}\right)\left(H_{2}^{2}-H_{1}^{2}\right)^{2}+\frac{G^{2}}{4}\left(H_{1}^{2} H_{2}^{2}+T^{2} H_{1}^{2}+T^{2} H_{2}^{2}\right)+\frac{B^{2}}{2}\left(T^{2}+T^{\prime 2}\right)+\frac{G B t^{\prime} H_{1} H_{2}}{2 \sqrt{2}} \\
+V_{\text {soft }}\left(H_{1}, H_{2}\right)
\end{gathered}
$$

Because of the term linear in $T^{\prime}, T^{\prime}$ acquires a vacuum expectation value:

$$
\left\langle T^{\prime}\right\rangle=-\frac{G a_{1} a_{2}}{2 B} \equiv t^{\prime}
$$

In addition to the superpotential we can have an R-invariant soft-term in the Lagrangian of the form

$$
\mathcal{L}_{\text {soft }}=A \tilde{T}^{\prime a} \lambda^{a}
$$

where the $\lambda$ 's are the fermionic partner of the gauge bosons, i.e. the gauginos, and $\tilde{T}^{\prime}$ is the fermionic component of the superfield $\hat{T}^{\prime}$. 
The $\rho$ parameter,

$$
\rho=\frac{M_{W}^{2}}{M_{Z}^{2} \cos ^{2} \theta_{W}}
$$

is given at tree level by

$$
\rho=\frac{\sum_{i} v_{i}^{2}\left[I(I+1)-\frac{Y^{2}}{4}\right]}{\sum_{i} v_{i}^{2} \frac{Y^{2}}{2}}
$$

where I is the weak isospin and $\mathrm{Y}$ is the hypercharge of the scalar multiplet. The $\rho$ parameter for our case, with a triplet and two Higgs doublets, is given by:

$$
\rho=\frac{v_{1}^{2}+v_{2}^{2}+4 t^{\prime 2}}{v_{1}^{2}+v_{2}^{2}}=1+\frac{4 t^{\prime 2}}{v_{1}^{2}+v_{2}^{2}}
$$

Since the $\rho$ parameter is known to equal 1 to within about $1 \%$, the vev of the triplet must be of the order of $12 \mathrm{GeV}$ or less.

The fermion mass matrix, which is our principal concern, divides into two charged matrices, each $2 \times 2$, and a neutral $3 \times 3$ matrix. To avoid phenomenological difficulties we require that the lightest eigenvalue of each of the charged mass matrices is more than half the $Z$ mass. The lightest neutral should either have mass greater than about half the $Z$ mass, or should couple sufficiently weakly to the $Z$ that it does not give too large a contribution to the $Z$ width. We have examined various ranges of parameters, and found that it is possible to satisfy all of these constraints. The constraint on the charged masses is easy to satisfy. It is more difficult to avoid light neutral particles. Indeed, study of the mass matrix reveals that the lightest neutral is never more massive than $\sin \left(\theta_{W}\right) M_{Z}$; this bound is saturated for $A, B \gg M_{W}$ and $G \gg g, g^{\prime}$. For example,

$\begin{array}{lllll}A & B & G & v_{1} / v_{2} & m_{l} \\ 9000 & 900 & 1 & 1 & 44 \\ 900 & 900 & 10 & .01 & 44\end{array}$

On the other hand, for a wide range of parameters, this light state contains nearly equal admixtures of the two Higgsinos, $\tilde{H}_{1}$ and $\tilde{H}_{2}$. Because these fields couple to the $Z$ with opposite signs, the coupling of this particle to the $Z$ is suppressed. 


\section{Conclusions}

There are a number of lessons to be drawn from this work. First, it does not seem so difficult to build models in which supersymmetry is dynamically broken. The price one pays is the introduction of light states beyond those of the minimal supersymmetric standard model. In addition, one requires that hypercharge be unified within a larger group. Needless to say, it is not clear whether such an approach will fit neatly into conventional grand unification or string theory.

We have also commented on some ideas of Hall and Randall for constructing theories with unbroken $\mathrm{R}$ symmetries. These have the potential to solve some of the other naturalness problems of supersymmetric theories. We have noted that in the context of supergravity, it is difficult to understand both vanishing cosmological constant and the existence of $\mathrm{R}$ symmetries. Ignoring this question, we have considered various aspects of these theories, and have noted that it may be necessary to add light triplets in the low energy theory to obtain phenomenologically viable models. We have seen that models of this type almost always yield new, relatively light fermions with interesting properties.

It is perhaps of interest to comment on one other set of naturalness issues as we close this paper. Cosmological and astrophysical constraints suggest that the axion decay constant is in the range $10^{11}-10^{12} \mathrm{GeV}$. Since this scale is similar to the scale $M_{\text {int }}$, it is natural to ask whether these two scales might be related. Indeed, this possibility was suggested some time ago by $\mathrm{Kim}^{[8]}$ and its possible cosmological significance has been considered by Rajagopal, Turner and Wilczek. ${ }^{[9]}$ However, while this coincidence is tantalizing, it is also problematic. In the models considered by Kim, for example, the axion couples to quarks with masses of order $M_{\text {int }}$. The axion, however, also couples (with dimensionless couplings) to the 'Goldstino' (the longitudinal component of the gravitino). As a result, in this model, there are diagrams at three loop order which involve only dimensionless couplings and give mass to squarks. These masses are of order

$$
m_{\tilde{q}}^{2} \sim \frac{\alpha_{s}{ }^{2}}{\pi} \frac{\lambda^{2}}{16 \pi^{2}} M_{i n t}^{2}
$$

Here $\lambda$ describes the coupling of the axino multiplet to the goldstino multiplet. 
Unless $\lambda$ is extremely small $\left(\lambda<10^{-6}\right.$ or so), squarks will obtain unacceptably large masses.

This problem appears quite general. In order to link supersymmetry breaking directly with the axion, the axion multiplet must couple to the goldstone multiplet. On the other hand, in order to have the correct coupling to $F \tilde{F}$, the axion must couple to fields carrying color. But this means that the hidden sector is not really hidden; while gauge fields may only couple to the hidden sector through loops, these couplings are not suppressed by factors of $\frac{1}{M_{P}}$. There is, of course, no problem in simply introducing the axion multiplet separately, with no (dimensionless) couplings to the hidden sector. However, in this case one needs some other way to understand the coincidence of scales. ${ }^{[16]}$

\section{Acknowledgements}

We wish to thank L. Hall and L. Randall for conversations about their work. We especially wish to thank Vadim Kaplunovsky for discussions of problems associated with anomalies, and for suggesting that various non-supersymmetric results even in apparently finite calculations could result from a need for regularization. This work supported in part by DOE contract DE-AC02-83ER40107.

\section{REFERENCES}

1. M. Dine, A. Kagan and S. Samuel, Phys. Lett. 243B (1990) 250.

2. I. Affleck, M. Dine, N. Seiberg, Nucl. Phys. B241 (1984) 493.

3. I. Affleck, M. Dine, N. Seiberg, Phys. Rev. Lett. 137B (1984) 187.

4. I. Affleck, M. Dine, N. Seiberg, Nucl. Phys. B256 (1984) 557.

5. L. Hall and L. Randall, Nucl. Phys. B352 (289) 1991.

6. J. Polchinski and L. Susskind, Phys. Rev. D26 (1982) 3661.

7. L. Alvarez-Gaume, J. Polchinski, and M. Wise, Nucl. Phys. B221 (1983) 495.

8. J. Kim, Phys. Lett. 136B (1984) 378.

9. K. Rajagopal, F. Wilczek, W. Turner, Nucl. Phys. B358 (1991), 447.

10. E. Witten, Nucl. Phys. B188 (1981) 513.

11. M.J. Grisaru, W. Siegel, and M. Rocek, Nucl. Phys. 159 (1979) 429.

12. E. Witten, Nucl. Phys. B202 (1982) 253. 
13. E. Cremmer, B. Julia, J. Scherk, S. Ferrara, L. Girardello and P. van Niewenhuizen, Nucl. Phys. B147 (1979) 105; J. Wess and J. Bagger, Supersymmetry and Supergravity, Princeton University Press, Princeton (1981) and Johns Hopkins preprint (1990).

14. H. Haber and R. Hempfling, Phys. Rev. Lett. 66 (1991) 1815; J. Ellis, G. Ridolfi and F. Zwirner, Phys. Lett. B257 (1991) 83.

15. L. Hall and L. Randall, private communication.

16. M. Dine, Talk at the Cincinnati Symposium in honor of Louis Witten, to appear. 